\title{
Q1 Variabilidade espacial da biomassa da forragem e taxa de lotação animal em pastagem de capim Marandu
}

Sabino Pereira da Silva Neto ${ }^{1}$

Antonio Clementino dos Santos ${ }^{2}$

Rafael Nunes Garcia ${ }^{3}$

João Lucas Aires Dias ${ }^{4}$

Áyzik Macedo Silva ${ }^{5}$

Paulo Alexandre Rodrigues Pereira ${ }^{6}$

\section{Resumo}

O objetivo deste estudo foi realizar a modelagem variográfica da disponibilidade de matéria seca da Urochloa brizantha cv. Marandu e a simulação da taxa de lotação animal por meio do ajuste dos modelos esférico, exponencial e gaussiano ao semivariograma experimental, bem como a robustez das predições. A biomassa da gramínea foi coletada em 50 pontos em uma área de 36,22 ha. A simulação da taxa de lotação foi realizada com base na disponibilidade de folhas verdes em cada ponto amostrado, consumo diário de matéria seca por cada unidade animal (UA) e o tempo de pastejo. Os dados referentes às variáveis foram submetidos à análise descritiva, estudo geoestatístico e interpolação por krigagem ordinária. A modelagem variográfica da disponibilidade de matéria seca do capim marandu e a taxa de lotação foram caracterizadas pelos modelos esférico, exponencial e gaussiano. Entretanto, apesar da aparente precisão dos ajustes, o modelo esférico apresentou melhor inferência, segundo o critério de informação de Akaike e soma dos erros ao quadrado. Assim, a adoção de modelos com ajustes de critérios somente visuais levam a estimativas da disponibilidade de biomassa de forragem e da taxa de lotação animal que não refletem a realidade da área.

Palavras-chave: Estrutura do pasto. Carga animal. Distribuição espacial. Pecuária de precisão. Produção animal. Semivariograma.

\footnotetext{
1 Instituto Federal do Tocantins, docente pesquisador. Gurupi, Tocantins, Brasil. sabino.pereira@ifto.edu.br. Alameda Madrid, 545, Jardim Sevilha, Gurupi, TO, CEP: 77410-470.

2 Universidade Federal do Tocantins, docente pesquisador. Araguaína, Tocantins, Brasil. clementino@mail.uft.edu.br. BR 153, Km 112, Fazenda Universitária, Araguaína, TO, CEP: 77804-970.

3 Instituto Federal do Tocantins, discente do ensino do médio integrado ao agronegócio. Gurupi, Tocantins, Brasil.rafael.nunes.g@hotmail.com. Alameda Madrid, 545, Jardim Sevilha, Gurupi, TO, CEP: 77410-470.

4 Instituto Federal do Tocantins, discente do ensino médio integrado ao agronegócio. Gurupi, Tocantins, Brasil. lucassingle@hotmail.com. Alameda Madrid, 545, Jardim Sevilha, Gurupi, TO, CEP: 77410-470.

5 Instituto Federal do Tocantins, discente do ensino médio integrado ao agronegócio. Gurupi, Tocantins, Brasil. ayzik83@hotmail.com. Alameda Madrid, 545, Jardim Sevilha, Gurupi, TO, CEP: 77410-470.

6 Instituto Federal do Tocantins, discente do ensino médio integrado ao agronegócio. Gurupi, Tocantins, Brasil. rodriguespereira23@gmail.com. Alameda Madrid, 545, Jardim Sevilha, Gurupi, TO, CEP: 77410-470.
} 


\section{Introdução}

A eficiência do sistema de produção animal em pastagens é ditada em especial pelo crescimento, utilização e conversão da gramínea em produto animal (HODGSON, 1990). Assim, é necessário compreender a estrutura do pasto e sua relação com a forragem produzida e consumida pelos animais para fundamentar ações de manejo que possam ser racionais e sustentáveis (GONÇALVES et al., 2009). A estrutura da pastagem é usualmente definida como a distribuição espacial da biomassa da gramínea em uma determinada área (CARVALHO et al., 2001).

Os sistemas de produção de bovinos em pastagens no Brasil utilizam predominantemente 0 método de pastejo sob lotação contínua (SANTOS et al., 2014). Esse sistema de manejo da pastagem em comparação à lotação intermitente promove maior heterogeneidade do pastejo (BARTHRAM et al., 2005), gerando variabilidade espacial na disponibilidade da forragem. Além disso, os fatores intrínsecos às características edafoclimáticas fazem com que a vegetação existente na pastagem seja heterogênea.

A caracterização dos padrões espaciais da disponibilidade da forragem, bem como das taxas de lotação em determinada área de produção de gramínea, pode beneficiar a tomada de decisões de manejo da pastagem de modo a criar estratégias para que os animais pastejem em locais onde tenha maior oferta de forragem. Para tanto, os métodos que caracterizam a estrutura horizontal do pasto devem ser investigados.

A heterogeneidade do pasto pode ser inferida por meio da modelagem dos padrões espaciais e os valores podem ser estimados em locais não observados ou amostrados. Para isso, a estrutura ou dependência espacial do pasto deve ser representada pelo semivariograma, que é a ferramenta central da geoestatística. Apesar disso, nem sempre os semivariogramas são robustos ou precisos devido à influência do número de amostras e à escolha do modelo que melhor se ajuste aos dados da semivariância experimental. Gross Filho et al. (2013) observaram que a estrutura espacial das características agronômicas do capim marandu podem ser preditas quando se utilizam no mínimo 120 pontos amostrais. Entretanto, não foram investigadas as diferenças entre os modelos ajustados ao semivariograma experimental da variabilidade espacial da gramínea.

Assim, o objetivo deste estudo foi realizar a caracterização espacial da disponibilidade de matéria seca e a taxa de lotação animal em pastagem de Urochloa brizantha cv. Marandu por meio do ajuste dos modelos esférico, exponencial e gaussiano ao semivariograma experimental, bem como a robustez dessas predições.

\section{Material e métodos}

O local do estudo está situado entre as latitudes $11^{\circ} 31^{\prime} 41^{\prime \prime}$ S e $11^{\circ} 30^{\prime} 54^{\prime \prime}$ S e longitudes $47^{\circ} 01^{\prime} 33^{\prime \prime}$ W e $47^{\circ} 01^{\prime}$ 8" W, na Chácara Nossa Senhora Aparecida, município de Dianópolis - Tocantins. A área de pastagem de Urochloa brizantha cv. Marandu utilizada no experimento foi estabelecida no ano de 2008 e mantida sob lotação contínua. Em março de 2014, foram demarcados 50 pontos de coleta de forragem (Figura 1) nessa área. Em cada ponto de amostragem pré-estabelecido, realizou-se a coleta da gramínea com quadro de amostragem de 0,5 m² (0,5 x $1 \mathrm{~m})$. A gramínea foi colhida à altura de $15 \mathrm{~cm}$ do solo e colocada em sacos identificados e levados para pesagem. Em seguida, os sacos foram levados à estufa para secagem a $65^{\circ} \mathrm{C}$ por 72 horas. Após secagem as amostras foram pesadas e seus pesos foram convertidos para disponibilidade de matéria seca da gramínea expressa em $\mathrm{g} \mathrm{m}^{-2}$. 


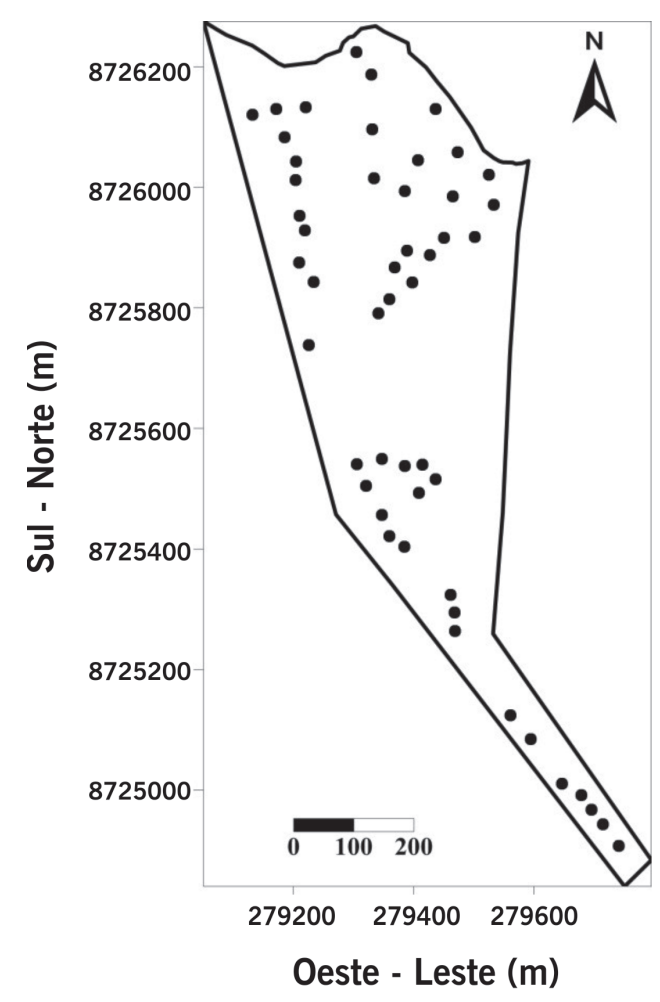

Figura 1. Locais de coleta da Urochloa brizantha cv. Marandu.

Fonte: Elaboração dos autores

A simulação da taxa de lotação foi realizada com base na disponibilidade de folhas verdes em cada ponto amostrado, consumo diário de matéria seca por cada unidade animal (UA) e o tempo de pastejo. Diante disso, estimou-se que uma UA necessita de $8 \%$ de oferta de matéria seca de folhas verdes para consumir $2 \%$ para cada quilograma de peso vivo, o tempo de pastejo é de 10 dias e cada UA corresponde a $450 \mathrm{~kg}$ de peso vivo. Para tanto, foi utilizada a seguinte equação:

Carga Animal $(\mathrm{kg} / \mathrm{ha})=\frac{\text { Disponibilidade de matéria seca de folhas verde } \times 100}{\text { Tempo de pastejo } \times \text { Oferta de folhas }}$

Taxa de lotação (UA/ha) $=\frac{\text { Carga Animal }}{450}$

Os dados da disponibilidade de matéria seca e taxa de lotação foram submetidos à análise exploratória, calculando-se a média, mediana, mínimo, máximo e os coeficientes de assimetria, curtose e variação; a normalidade de distribuição dos dados foi testada.

A dependência espacial entre as observações foi caracterizada por meio do semivariograma estimado pela seguinte equação:

$\gamma=\frac{1}{2 \mathrm{~N}(\mathrm{~h})} \sum_{\mathrm{i}=1}^{\mathrm{N}(\mathrm{h})}\left[\mathrm{Z}\left(\mathrm{x}_{\mathrm{i}}\right)-\mathrm{Z}\left(\mathrm{x}_{\mathrm{i}}+\mathrm{h}\right)\right]^{2}$

Em que $\gamma$ é a semivariância, $N(h)$ o número de pares de valores experimentais medidos em $\left[Z\left(x_{i}\right), Z\left(x_{i}+h\right)\right]$, separados pela distância h. No presente estudo, os valores de $Z$ são a disponibilidade de matéria seca e a taxa de lotação animal, enquanto os valores de $x_{i}$ e $x_{i}+h$ foram definidos de acordo com a localização geográfica das amostras realizadas na área de pastejo. 
Neste trabalho foram testados os seguintes modelos de semivariograma: (a) esférico, $\gamma(\mathrm{h})=\mathrm{C}_{0}$ $+C_{1}\left[1,5(h / A)-0,5(h / A)^{3}\right]$ para $(0<h \leq A)$ e $\mathrm{y}(h)=C_{0}+C_{1}$ para $h>A$; (b) exponencial, $y(h)$ $=C_{0}+C_{1}[1-\exp (-h / A)]$; e (c) gaussiano, $\gamma(h)=C_{0}+C_{1}\left[1-\exp \left(-h^{2} / A^{2}\right)\right]$ em que "d" é a distância máxima para qual o semivariograma é definido e " $\mathrm{A}$ " o alcance. $\mathrm{O}$ ajuste dos semivariogramas possibilitou definir os valores do efeito pepita $\left(C_{0}\right)$, do alcance $(A)$ e do patamar $\left(C+C_{0}\right)$.

Para avaliação da escolha do modelo ajustado dos semivariogramas, utilizou-se do critério de informação de Akaike (AIC) e da soma dos erros ao quadrado (SSE). O critério de Akaike é dado pela seguinte expressão: $A I C=-2 \log L+2 K$, em que $L$ é a verossimilhança maximizada pelo modelo candidato e $\mathrm{K}$ é o número de parâmetros do modelo considerado.

A interpolação dos valores foi realizada por meio da krigagem ordinária, de modo a definir o padrão espacial da disponibilidade de matéria seca da gramínea e da taxa de lotação, o que permitiu a elaboração dos mapas de contorno utilizando o programa Surfer 8.0 com o auxilio da equação:

$\mathrm{Z}^{*}\left(\mathrm{x}_{0}\right)=\sum_{\mathrm{i}=1}^{\mathrm{N}} \lambda_{\mathrm{i}} \mathrm{Z}\left(\mathrm{x}_{\mathrm{i}}\right)$

Em que $Z^{*}\left(x_{0}\right)$ é o estimador de kirgagem; $\lambda_{i}$ é o peso da i-ésima localidade vizinha; $Z\left(x_{i}\right)$ é o valor da variável para a i-ésima localidade; $\mathrm{N}$ é o número de localidades vizinhas empregadas para interpolação do ponto.

A criação dos mapas de contorno permite compreender a variabilidade espacial da disponibilidade de matéria seca e a taxa de lotação animal na pastagem de capim marandu a partir dos modelos ajustados ao semivariograma experimental. Destarte, é possível visualizar e entender as diferenças práticas de manejo da pastagem que cada modelagem variográfica pode gerar.

\section{Resultados e discussão}

A estatística descritiva da disponibilidade de matéria seca da forragem e da taxa de lotação animal é apresentada na Tabela 1. Pode-se observar que o mínimo e máximo apresentam valores distantes, o que indica que a área tem elevada heterogeneidade dos dados. 0 grau de heterogeneidade horizontal do pasto pode ser descrito pelo coeficiente de variação (CV) (HIRATA, 2002). O valor do coeficiente de variação (CV), classificado como alto por Warrick e Nielsen (1980), confirma essa variabilidade. A média e mediana também apresentam valores relativamente distantes, o que tendencia os dados à distribuição assimétrica. Dessa forma, o teste de Shapiro-Wilks mostra que as variáveis analisadas nesta investigação científica não apresentam distribuição normal dos dados.

Tabela 1. Estatística descritiva da disponibilidade de matéria seca da forragem ( $\mathrm{g} \mathrm{m}^{-2}$ ) e da taxa de lotação animal (UA ha-1) em pastagem de Urochloa brizantha cv. Marandu.

\begin{tabular}{|c|c|c|c|c|c|c|c|c|}
\hline \multirow{2}{*}{ Variável } & \multirow{2}{*}{ Mínimo } & \multirow{2}{*}{ Média } & \multirow{2}{*}{ Mediana } & \multirow{2}{*}{ Máximo } & \multicolumn{3}{|c|}{ Coeficiente } & \multirow{2}{*}{$p$-valor ${ }^{1}$} \\
\hline & & & & & Variação & Curtose & Assimetria & \\
\hline $\mathrm{DMS}^{2}$ & 5,40 & 49,91 & 26,24 & 140,28 & 63,00 & $-0,13$ & 0,55 & $0,01^{* *}$ \\
\hline $\mathrm{TLA}^{3}$ & 0,15 & 1,38 & 0,73 & 3,90 & 63,00 & $-0,13$ & 0,55 & $0,02^{* *}$ \\
\hline
\end{tabular}

${ }^{(1)}$ : teste de normalidade de Shapiro-Wilks; ${ }^{(*)}$ : significativo a $5 \%,{ }^{(*)}$ : não-significativo a $5 \%$; ${ }^{(2)}$ : Disponibilidade de matéria seca; ${ }^{(3)}$ : Taxa de lotação animal.

Fonte: Elaboração dos autores

Os altos valores de CV já foram observados em estudo da distribuição espacial da gramínea em pastagens de Urochloa brizantha cv. Marandu (SILVA NETO et al., 2012). Apesar do CV demonstrar a ocorrência de certo grau de heterogeneidade do pasto, esse não é capaz de descrever como isso 
ocorre no plano horizontal da pastagem, nem de estimar possíveis locais onde os animais poderiam pastejar em função dessa variação espacial. Diante disso, a modelagem variográfica é importante para caracterizar espacialmente a disponibilidade de forragem e a lotação animal de uma pastagem, pois os padrões de deslocamento e procura dos animais em pastejo são afetados pela estrutura do pasto (GONÇALVES et al., 2009). Sob essa ótica, conjectura-se que é inoportuno o uso de valores médios para discriminar os padrões espaciais de pastagens, pois esses não permitem estimar os padrões de pastejo e de desempenho dos animais.

Apesar da ausência de distribuição normal dos dados, os coeficientes de curtose e assimetria são próximos a zero, o que demonstra que a distribuição dos dados não apresenta caudas muito alongadas que possam comprometer os resultados do ajuste dos modelos ao semivariograma experimental (SILVA et al., 2010). Logo, observa-se nas Figuras 2 e 3 que os modelos teóricos ajustados apresentam patamares bem definidos. Nesse sentido, a construção do gráfico do semivariograma experimental demonstra o ajuste sobre os pontos de uma função contínua, ou seja, modelo teórico que descreve o comportamento dos dados no espaço (OLIVER, 2010). Matematicamente é obrigatório que o modelo ajustado seja monótono, crescente e tenha positividade condicional definida (WEBSTER; OLIVER, 2007).

A. Esférico

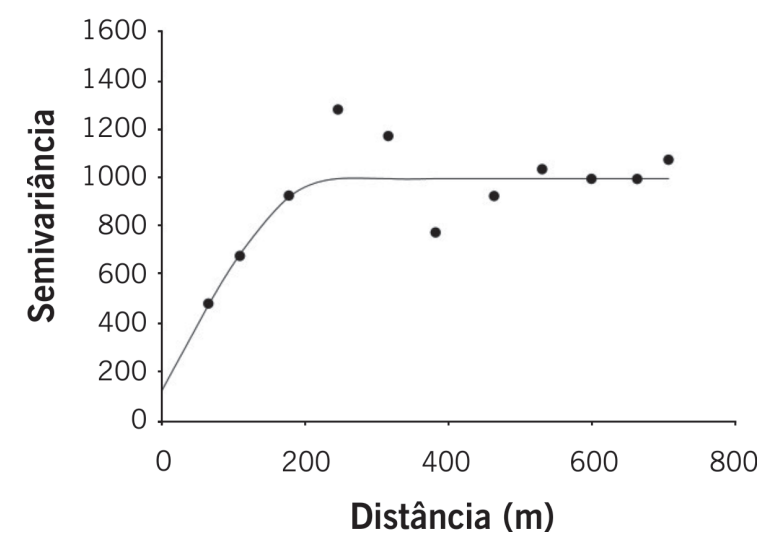

B. Exponencial

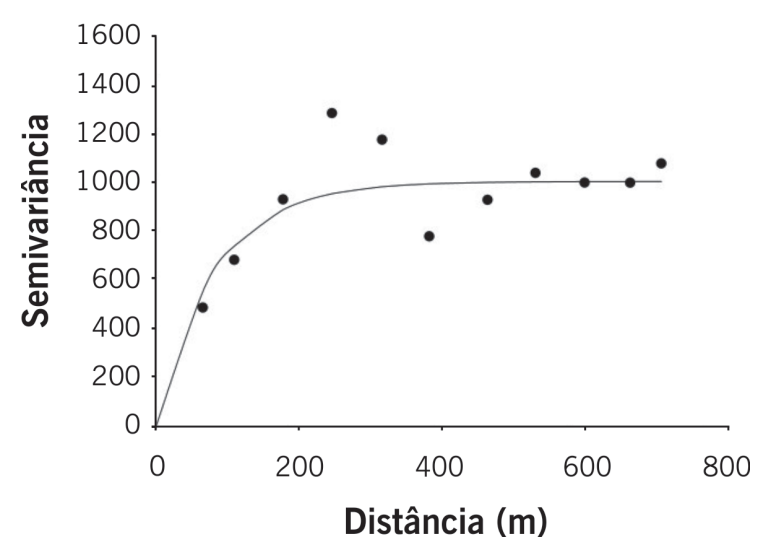

\section{Gaussiano}

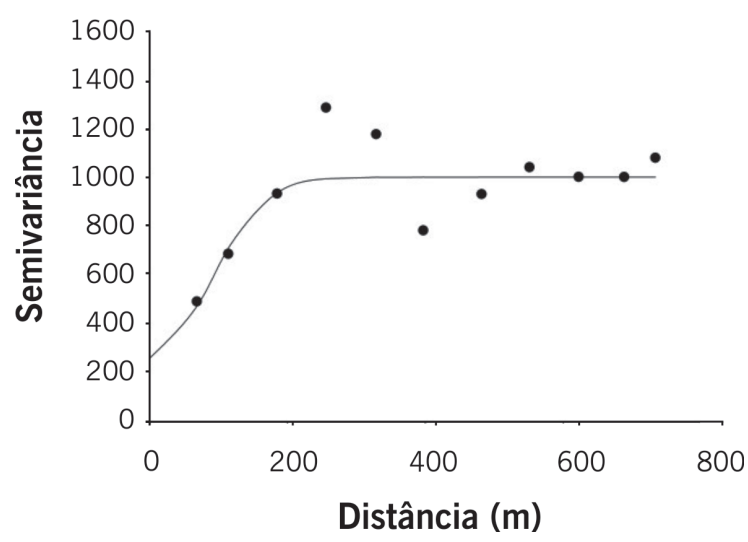

Figura 2. Semivariogramas ajustados aos valores de disponibilidade de matéria seca em pastagem de Urochloa brizantha cv. Marandu.

Fonte: Elaboração dos autores 
A. Esférico

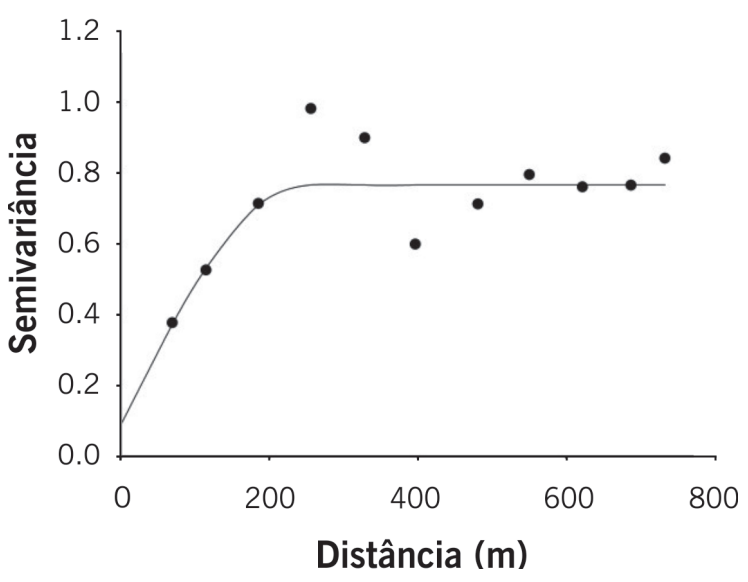

B. Exponencial

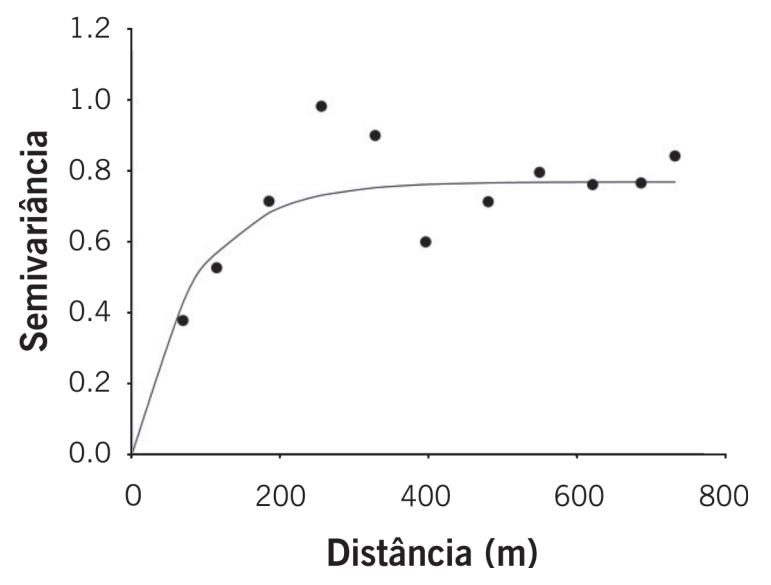

C. Gaussiano

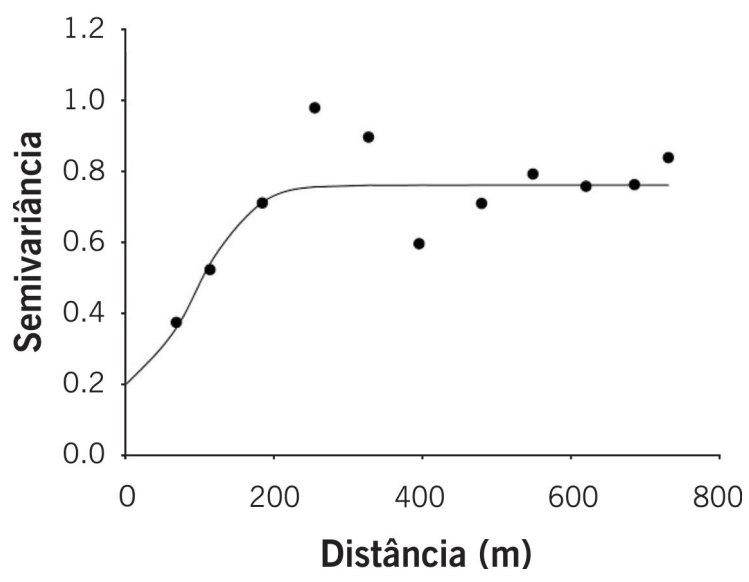

Figura 3. Semivariogramas ajustados aos valores de taxa de lotação animal em pastagem de Urochloa brizantha cv. Marandu.

Fonte: Elaboração dos autores

A distância máxima usada para construção dos semivariogramas foi de 800 metros e representa $50 \%$ da diagonal da área de estudo. Os modelos ajustados ao semivariograma empírico foram o esférico, exponencial e gaussiano; esses, aparentemente, poderiam estimar a variabilidade espacial da disponibilidade de biomassa da forrageira e a taxa de lotação animal na pastagem de capim Marandu. 0 ajuste do modelo teórico é etapa importante do estudo variográfico, tendo em vista que o estimador linear (krigagem) depende do valor do modelo do semivariograma para cada distância especificada (VIEIRA et al., 1981). 0 ajuste espacial pode ser feito de maneira visual, ou seja, sem nenhum procedimento matemático; no entanto, esse é mais sujeito a erros (LANDIM, 2006). Ademais, existem métodos que atenuam ou até mesmo retiram a subjetividade das estimativas dos parâmetros do semivariograma. Entre os métodos disponíveis, destacam-se os métodos dos quadrados mínimos ordinários e quadrados mínimos ponderados e o método da máxima verossimilhança (MELLO et al., 2005). Esses métodos de ajuste podem ser avaliados por meio das técnicas de validação cruzada e preditiva (WEBSTER; OLIVER, 2007) e do critério de informação de Akaike (AIC) (AKAIKE, 1973). 
Na Tabela 2 podem-se observar os parâmetros dos modelos ajustados aos semivariogramas. Nesse sentido, tanto para a biomassa de forragem disponível quanto para a taxa de lotação animal, o modelo esférico apresentou menor valor do AIC e soma dos erros ao quadrado (SSE) com valores quase idênticos aos do modelo gaussiano. Por outro lado, o modelo exponencial teve maiores valores de AIC e SSE, o que indica que esse modelo pode gerar estimativas que não representam a realidade da área investigada.

Tabela 2. Modelos e parâmetros estimados dos semivariogramas ajustados à disponibilidade de matéria seca da forragem $\left(\mathrm{g} \mathrm{m}^{-2}\right)$ e taxa de lotação animal (UA ha-1) em pastagem de Urochloa brizantha cv. Marandu.

\begin{tabular}{ccccccc}
\hline \multirow{2}{*}{ Variável } & \multirow{2}{*}{ Modelo } & \multicolumn{3}{c}{ Parâmetro } & \multirow{2}{*}{ SSE $^{4}$} & \multirow{2}{*}{ AIC $^{5}$} \\
\cline { 3 - 5 } & & $\mathbf{C O}_{1}$ & $\mathbf{C O}+\mathbf{C}_{2}$ & $\mathbf{A}^{3}$ & & \\
& Esférico & 128,60 & 860,30 & 246,50 & $16,51 \times 10^{4}$ & 138,20 \\
DMS $^{6}$ & Exponencial & 0,00 & 992,00 & 85,15 & $20,45 \times 10^{4}$ & 140,50 \\
& Gaussiano & 255,80 & 730,50 & 118,20 & $17,12 \times 10^{4}$ & 138,60 \\
\hline \multirow{3}{*}{ TLA $^{7}$} & Esférico & 0,09 & 0,66 & 245,90 & 0,10 & $-19,22$ \\
& Exponencial & 0,00 & 0,76 & 84,96 & 0,12 & $-16,91$ \\
& Gaussiano & 0,20 & 0,56 & 117,90 & 0,10 & $-18,92$ \\
\hline
\end{tabular}

${ }^{(1)} \mathrm{C}_{0}$ : efeito pepita; ${ }^{(2)} \mathrm{C}_{0}+\mathrm{C}$ : patamar; ${ }^{(3)} \mathrm{A}$ : alcance; ${ }^{(4)} \mathrm{SSE}$ : raiz quadrada do erro quadrático médio; ${ }^{(5)} \mathrm{AIC}$ : Critério de Akaike; ${ }^{(6)}$ : Disponibilidade de matéria seca; ${ }^{(7)}$ : Taxa de lotação animal.

Fonte: Elaboração dos autores

De acordo com Paula Neto et al. (2014), a distribuição espacial de massa seca de Urochloa híbrida cv. Mulato II ajustou-se, na maioria dos casos, ao modelo exponencial. Em contrapartida, Grego et al. (2013) observaram que a biomassa de Urochloa brizantha, no verão e outono, ajusta-se ao modelo esférico. Em vista disso, ressalta-se que o mais importante não é o modelo ajustado ao semivariograma e sim a exatidão do modelo em caracterizar a distribuição espacial do atributo investigado na pastagem. Apenas com estimativas robustas o uso das ferramentas da geoestatística seria justificável em comparação ao uso de valores médios que constantemente são usados para caracterizar a estrutura horizontal do pasto.

Apesar de os valores de AIC e SSE dos modelos esférico e gaussiano estarem próximos, os valores de efeito pepita, patamar e alcance foram diferentes devido às características matemáticas de cada um deles (Tabela 2). Entretanto, os alcances foram bem maiores que o modelo exponencial, o que pode ter influências sobre as estimativas geradas na interpolação dos dados. Isso porque a krigagem ordinária usa o alcance de um atributo como indicativo, o qual garante que todos os pontos dentro de um círculo de determinado raio sejam tão similares que podem ser usados para estimar valores para qualquer ponto entre eles (MACHADO et al., 2007). Assim, alcances maiores, a depender da grade de amostragem, permitem o uso de um número maior de pontos para estimar outro ponto qualquer dentro desse círculo.

A partir dos ajustes dos modelos ao semivariograma experimental para as variáveis do estudo, estimaram-se os valores para locais não amostrados por meio do método de interpolação de krigagem ordinária. Com esses valores estimados, foi possível construir mapas de contorno das variáveis em função do modelo utilizado no ajuste. As superfícies de krigagem das Figuras 4 e 5 ilustram os padrões gerais dos semivariogramas da disponibilidade de biomassa da gramínea e simulação de taxa de lotação, discutidos anteriormente, permitindo visualizar os arranjos da distribuição espacial na área investigada. 


\section{A. Esférico}

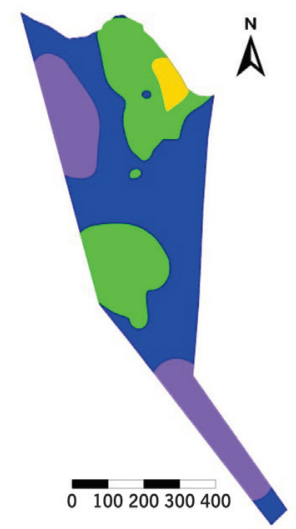

A. Exponencial

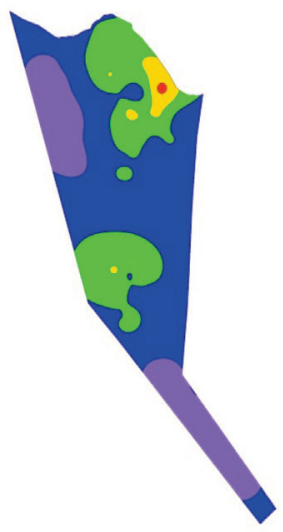

\section{Gaussaniano}

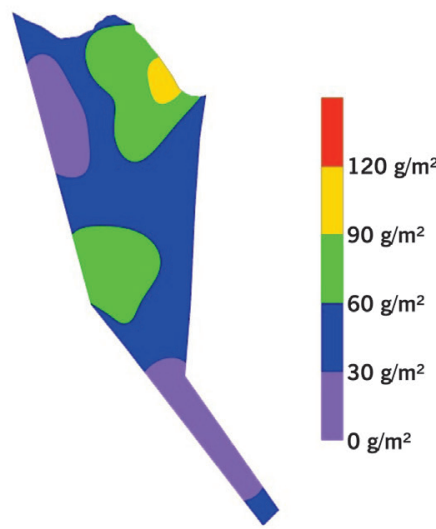

Figura 4. Mapas representativos da disponibilidade de matéria seca $\left(\mathrm{g} \mathrm{m}^{-2}\right)$ em pastagem de Urochloa brizantha cv. Marandu em função do ajuste ao modelo esférico (A), exponencial (B) e gaussiano (C).

Fonte: Elaboração dos autores

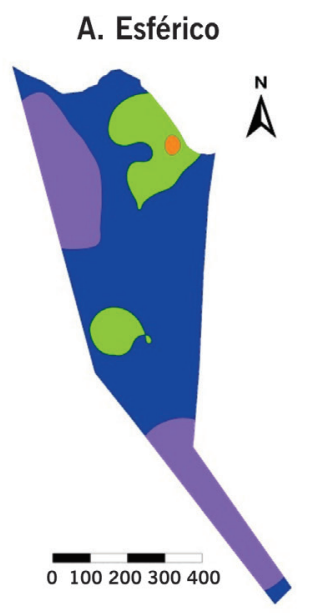

A. Exponencial

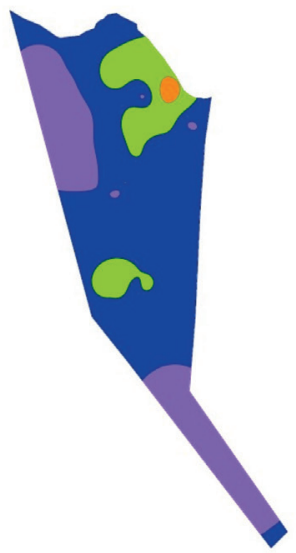

C. Gaussaniano

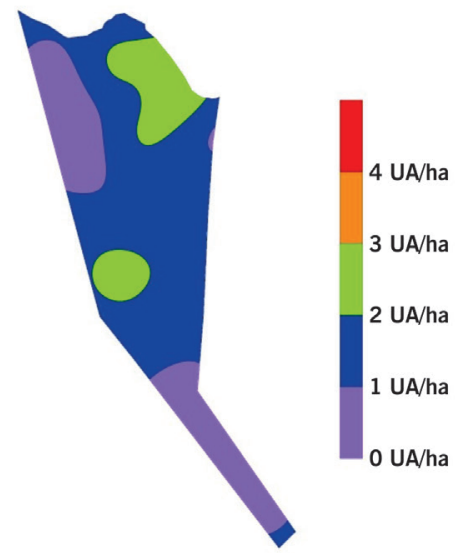

Figura 5. Mapas representativos da taxa de lotação animal (UA ha-1) em pastagem de Urochloa brizantha cv. Marandu em função do ajuste ao modelo esférico (A), exponencial (B) e gaussiano (C).

Fonte: Elaboração dos autores

Os arranjos espaciais apresentados (Figuras 4 e 5) podem permitir aprender e compreender como os animais modificam os padrões de pastejo, uma vez que esses são induzidos a alterar seu deslocamento por qualquer característica com dependência espacial, que podem também interferir no seu desempenho e eficiência de uso do recurso forrageiro (PÁSCOA; COSTA, 2007). Além disso, os ruminantes pastando em pastagens heterogêneas mudam suas estratégias de procura pelo alimento como tentativa de aumentar ou mesmo manter a eficiência de ingestão de forragem (MEZZALIRA et al., 2013). Apesar disso, eles ainda irão manter um pastejo desuniforme, ocasionando mudanças no plano horizontal que resulta na variabilidade espacial da vegetação do pasto (SANTOS et al., 2014).

Nas Figuras 4A, 4B e 4C observa-se que os mapas da disponibilidade de matéria seca do capim marandu são bem parecidos. Entretanto, pequenas diferenças podem ser notadas entre os mapas de cada modelo teórico. Para detectar essas diferenças nos mapas, realizou-se o cálculo da área da pastagem em cada intervalo ou classe de disponibilidade de matéria seca em função do modelo usado no ajuste (Tabela 3 ). 
Tabela 3. Classes de disponibilidade de matéria seca $\left(\mathrm{g} \mathrm{m}^{-2}\right)$ e suas respectivas áreas na pastagem de Urochloa brizantha cv. Marandu em função do modelo ajustado ao semivariograma experimental.

\begin{tabular}{cccc}
\hline CLASSES DE & \multicolumn{3}{c}{ MODELO } \\
\cline { 2 - 4 } DMS $^{(1)}$ & ESFÉRICO & EXPONENCIAL & GAUSSIANO \\
\hline$\left(\mathrm{G} \mathrm{M}^{-2}\right)$ & \multicolumn{1}{c}{ ÁREA DA PASTAGEM OCUPADA PELA CLASSE (HA) } \\
\hline $0-30$ & 7,40 & 7,08 & 7,16 \\
$30-60$ & 17,67 & 18,77 & 17,80 \\
$60-90$ & 10,25 & 9,25 & 10,46 \\
$90-120$ & 0,90 & 1,07 & 0,80 \\
$120-130$ & 0,00 & 0,06 & 0,00 \\
\hline Área Total (ha) & 36,22 & 36,22 & 36,22 \\
\hline
\end{tabular}

(1): Disponibilidade de matéria seca.

Fonte: Elaboração dos autores

As áreas de cada intervalo de classe da disponibilidade de MS nos modelos esférico e gaussiano, de modo geral, pouco se distanciaram. O modelo exponencial, a partir da classe de 30 a $60 \mathrm{~g} \mathrm{~m}^{-2}$, teve valores distintos dos outros modelos investigados. Lembrando que o ajuste do modelo exponencial da semivariância apresentou valores maiores de AIC e SSE, o que indicaria ao técnico não adotar esse modelo para criar mapas de predição da disponibilidade de MS da pastagem de capim Marandu.

Para disponibilidade de matéria seca, a variabilidade espacial da taxa de lotação animal predita para pastagem de capim marandu apresenta algumas diferenças que foram investigadas pela distinção do tamanho da área ocupada por cada classe de lotação em função do modelo ajustado ao semivariograma (Tabela 4). Os modelos esférico e gaussiano, de maneira geral, não se distinguiram tanto. 0 ajuste exponencial, nos intervalos de 1 a 2 UA ha-1, apresentou a maior diferença em relação aos demais modelos do estudo.

Tabela 4. Intervalos de taxa de lotação animal (UA ha-1) e suas respectivas áreas na pastagem de Urochloa brizantha cv. Marandu em função do modelo ajustado ao semivariograma experimental.

\begin{tabular}{cccc}
\hline \multirow{2}{*}{ INTERVALOS DE TLA } & \multicolumn{3}{c}{ MODELO } \\
\cline { 2 - 4 } & ESFÉRICO & EXPONENCIAL & GAUSSIANO \\
\hline (UA HA $\left.^{-1}\right)$ & 9,40 & ÁREA DA PASTAGEM OCUPADA PELA CLASSE (HA) \\
\hline $0-1$ & 21,73 & 8,97 & 9,10 \\
$1-2$ & 4,91 & 22,35 & 21,52 \\
$2-3$ & 0,18 & 4,60 & 5,60 \\
$3-4$ & 36,22 & 0,30 & 0,00 \\
\hline Área Total (ha) & 36,22 & 36,22 \\
\hline
\end{tabular}

(1): Taxa de lotação animal.

Fonte: Elaboração dos autores

Observa-se que a modelagem variográfica foi capaz de caracterizar a distribuição espacial da disponibilidade de biomassa seca e de possíveis taxas de lotação animal no ambiente pastoril. Vislumbra-se que o uso de ferramentas da geoestatística poderá auxiliar na compreensão da variabilidade horizontal do pasto e consequente predições de comportamento e desempenho dos animais em pastejo. Nessa ótica, as investigações que buscam conhecer como, quando e onde os herbívoros irão pastejar em pastagens heterogêneas (MEZZALIRA et al., 2013) terão subsídios científicos para esclarecer esses padrões de pastejo. 


\title{
Conclusão
}

A variabilidade espacial da matéria seca da forragem e da taxa de lotação animal em pastagem de Urochloa brizantha cv. Marandu foi caracterizada pelos modelos esférico, exponencial e gaussiano. Entretanto, apesar da aparente precisão dos ajustes, o modelo esférico apresentou melhor inferência segundo o critério de informação de Akaike e soma dos erros ao quadrado. Portanto, a adoção de modelos com ajustes com critérios somente visuais levam a estimativas da disponibilidade de biomassa de forragem e da taxa de lotação animal que não refletem a realidade da área.

\section{Agradecimentos}

Ao IFTO pelo apoia à pesquisa (EDITAL No 39/2014/REITORIA/IFTO) e ao CNPq pela concessão de bolsas na modalidade PIBIC-EM.

\section{Spatial variability of forage biomass and stocking rate in Marandu grass pasture}

\begin{abstract}
The objective of this study was to perform variogram modeling of the availability of dry matter of Urochloa brizantha cv. Marandu and to execute the simulation of stocking rate by adjusting the spherical, exponential and Gaussian models to the experimental semivariogram and the robustness predictions. The grass biomass was collected in 50 points in an area of 36.22 ha. The simulation of the stocking rate was based on the availability of green leaves at each sampled point, daily dry matter intake per animal unit and grazing time. The data relating to the variables were submitted to descriptive analysis, geostatistical study and interpolation by ordinary kriging. The variogram modeling of dry matter availability of Marandu grass and stocking rate were characterized by spherical, exponential and Gaussian models. However, despite the apparent accuracy of the adjustments, the spherical model showed better inference according to Akaike information and sum of squared errors. Thus, the adoption of models with adjustments of only visual criteria take to estimates of forage biomass availability and stocking rate that does not reflect the area reality.
\end{abstract}

Keywords: Pasture structure animal production. Spatial distribution. Precision livestock stocking rate. Semivariogram.

\section{Referências}

BARTHRAM, E.; DUFF, G. T. I.; ELSTON, D. A.; GRIFFITHS, J. H.; COMMON, T. G.; MARRIOTT, C. A. Frequency distributions of sward height under sheep grazing. Grass and Forage Science, v. 60, n. 1, p. 04-16, 2005.

CARVALHO, P. C. F.; RIBEIRO FILHO, H. M. N.; POLI, C. H. E. C.; MORAES, A.; DELAGARDE, R. Importância da estrutura da pastagem na ingestão e seleção de dietas pelo animal em pastejo. In: MATTOS, W.R.S. (Org.). Anais da XXXVIII REUNIÃO ANUAL DA SOCIEDADE BRASILEIRA DE ZOOTECNIA, Piracicaba, 2001, v. 1, p. 853-871. Disponivel em: http://www.ufrgs.br/gpep/docu- 
ments/capitulos/Import\%C3\%A2ncia\%20da\%20estrutura\%20da\%20pastagem\%20na\%20ingest\%C3\%A3o\%20e\%20sele\%C3\%A7\%C3\%A30\%20de\%20dietas\%20pelo\%20animal\%20em\%20 pastejo.pdf. Acesso em: 29 ago. 2014.

GONÇALVES, E. N.; CARVALHO, P. C. F.; DEVINCENZI, T.; LOPES, M. L. T.; FREITAS, F. K.; JACQUES, A. V. A. Relações planta-animal em ambiente pastoril heterogêneo: padrões de deslocamento e uso de estações alimentares. Revista Brasileira de Zootecnia. v. 38, n. 11, p. 2121-2126, 2009.

GREGO, C. R.; RODRIGUES, C. A. G.; NOGUEIRA, S. F.; GIMENES, F. M. A.; OLIVEIRA, A.; ALMEIDA, C. G. F.; FURTADO, A. L. S.; DEMARCHI, J. J. A. A. Variabilidade espacial do solo e da biomassa epígea de pastagem, identificada por meio de geostatística. Pesquisa Agropecuária Brasileira. v. 47, n. 9, p. 1404-1412, 2012.

GROSS FILHO, A. R.; SILVA NETO, S. P.; GARCIA, R. N.; SILVA, A. F.; MARTINS, N. T. L.; PENNO, M. S. Variabilidade espacial das características agronômicas da pastagem de Brachiaria brizantha cv. Marandu. In: JORNADA DE INICIAÇÃO CIENTíFICA E EXTENSÃO DO IFTO, 4, 2013, Paraíso. Anais...Paraíso: IFTO. Disponivel em: <http://propi.ifto.edu.br/ocs/index.php/jice/jice/paper/viewFile/5998/3126> Acesso em: 30 ago. 2014.

HIRATA, M. Herbage availability and utilisation in small-scale patches in a bahia grass (Paspalum notatum) pasture under cattle grazing. Tropical Grasslands, Austrália, v. 36, p. 13-23, 2002.

HODGSON, J. Grazing management: science into practice. London: Longman Group, 1990. 200p.

MACHADO, L. O.; LANA, A. M. Q.; LANA, R. M. Q.; GUIMARÃES, E. C.; FERREIRA, C. V. Variabilidade espacial de atributos químicos do solo em áreas sob sistema plantio convencional. Revista Brasileira de Ciência do Solo, Viçosa, v. 31, n. 3, p. 591-599, 2007.

MEZZALIRA, J. C.; BREMM, C.; TRINDADE, J. K. D.; GONDA, H. L.; VIEIRA, P. C.; CARVALHO, P. C. $\mathrm{F}$. Ingestive behaviour from the feeding station to patch level in heterogeneous environments. Journal of Animal Science Advances. v. 3, n. 12, p. 613-623, 2013.

PÁSCOA, A. G.; COSTA, M. J. R. P. Aplicação dos sistemas de informação geográfica para definição de estratégias de manejo de bovinos nas pastagens. Revista Brasileira de Zootecnia. v. 36, p. 45-51, 2007. Suplemento.

PAUlA NETO, J. J.; ALEXANDRINO, E.; SANTOS, A. C.; MENDES FILHO, G. O.; SILVA, D. P.; MELO, J. C. Distribuição espacial da altura do dossel e efeito sobre a cobertura do solo em pastos mantidos em lotação contínua. Bioscience Journal. v. 30, p. 650-658, 2014. Suplemento 2.

SANTOS, M. E. R.; GOMES, V. M.; FONSECA, D. M. Fatores causadores de variabilidade espacial do pasto de capim-Braquiária: manejo do pastejo, estação do ano e topografia do terreno. Bioscience Journal, Uberlândia, v. 30, n. 1, p. 210-218, 2014.

SILVA NETO, S. P.; SANTOS, A. C.; LIMA LEITE, R. L.; DIM, V. P.; NEVES NETO, D. N.; CRUZ, R. S. Dependência espacial em levantamentos do estoque de carbono em áreas de pastagens de Brachiaria brizantha cv. Marandu. Acta Amazônica. v. 42, n. 4, p. 547-556, 2012. 
WARRICK A. W.; NIELSEN, D. R. Spatial variability of soil physical properties in the field. In: HILLEL, D. (Ed.). Application of soil physics. New York: Academic Press, 1980.

\section{Histórico editorial}

Submetido em: 21/05/2015

Aceito em: 22/07/2015 